\title{
Literatur-Verzeichnis
}

\section{Geburtshülfe. Allgemeines.}

Albeck und Lohse, Ein Versuch, das Eklampaiegift auf experimentellem

Wege nachzuweisen. Zeitschr. f. Geb. u. Gyn Bd. 62. H. 1. S. 115. Chidichimo, F., La durata del periodo di riposo delle contrazioni dei

muscoli lisci. La ginecol. 15. März. S. 129. French, H., The Goulstonian lectures on the influence of pregnancy

upon certain medical diseases etc. Brit. med. Journ. 9. u. 16. Mai.

S. 1100 u. 1165. Henrich, C, Beitrag zur Geburt bei Doppelmissbildung der weiblichen Genitalien. Centralbl.. f. Gyn. 16. Mai. S. 658. v. Herff, C, Über die Bewertung gewisser Behandlungsmethoden der

Bakteriämien des Kindbettes, insbesondere der Hysterektomie.

Deutsche med. Wochenschr. 11. Juni. S. 1041. Jaks, A., Ein einfaches Hülfsmittel zur Erleichterung der Geburten.

Munch, med. Wochenschr. 26. Mai. S. 1135. Krönig, B., Schmerzlose Entbindungen im Dämmerschlaf. Deutsche

med. Wochenschr. 4. Juni. S. 993.

Literatur -Verzeichnis.

125

Lenk, H., Zur Frage der Stillfähigkeit. Munch, med. Wochenschr.

19. Mai. S. 1074. Mathes, P., Bee-bachtungen an mit Placentasaft durchströmten Hundenierßn. Zeitschr. f. Geb. u. Gyn. Bd. 62. H. 1. S. 108. Nagel, M., Über Stillpraxis und ihren Einfluss auf die Entwicklung des

Kindes. Munch, med. Wochenschr. 19. Mai. S. 1071. Piering, Über die Messung des graviden Uterus. Centralbl. f. Gyn.

23. Mai. S. 712. Schatz, F., Wann tritt die Geburt ein? Arch. f. Gyn. Bd. 85. H. 2.

S. 251. Sellheim, H., Die Entbindung durch die ,,Uterusbauchdeckenfistel”.

Centralbl. f. Gyn. 16. Mai. S. 641. Sfamení, P., Noch einige Worte über die Entstehung der Placenta

marginata und circumvallata. Centralbl. f. Gyn. 30. Mai. S. 737. Thomson, H., Klinische Erfahrungen über die Wirkung des Secacornin.

Centralbl. f. Gyn. 30. Mai. S. 742. Vezin, B., Sechs neue Fälle von Osteomalacie aus der Göttinger Univ.-

Frauenklinik. I.-D. Göttingen 1908.

Physiologi $\beta$ und Pathologic der Schwangßrschaft, der Geburt und des Wochenbettes.

Cálderini, Q., Su di alcune emorrhagie ostetriche. Lucina. 1. Mai.

S. 65. Freund, W. A., Über Wehenachwäche und ihre Bedeutung. Deutsche med. Wochenschr. 14. Mai. S. 857. Gräfenberg, E., Die prognostische Bedeutung der morphologißchen Blutelemente bei puerperalen Erkrankungen. Arch. f. Gyn. Bd. 85. 
H. 2. S. 302. Härle, Amylenhydrat bei Eklampsie. Munch, med. Wochenschr. 26. Mai.

S. 1134. v. Herff, Gemässigter oder radikaler Konservatismus in der Behandlung des engen Beckens. Munch, med. Wochenschr. 2. Juni. S. 1176. Hollmann, W., Ein Fall von Querlage mit drohendem Armvorfall bei

Uterus bicornis etc. St. Petersb. med. Wochenschr. No. 17. S. 172. Holzbach, E., Über Amaurose in der Schwangerschaft. Centralbl. f. Gyn.

23. Mai. S. 709. Meyer, A.W., Resultate der Nabelabklemmung. Wiener klin. Wochenschr.

7. Mai. S. 693. Möhlmann, K., Über die Therapie der Eklampsie. Zeitschr. f. Geb. u.

Gyn. Bd. 62. H. 1. S. 79. Müller, A., Zur Serumbehandlung des Puerperalfiebers. Munch, med.

Wochenschr. 19. Mai. S. 1069. Renter, W., Beiträge zur Behandlung der Eclampsia puerperalis. Inaug.-

Diss. Giessen. Mai. Rosenbach, H., Beitrag zur Frage der Entstehung der Schwangerschaft $\beta$ ödeme. I.-D. Göttingen 1908. Rosenfeld, V., Klinische Untersuchungen über die sogenannten Über-

drehungen des Kindes. Inaug.-Diss. Freiburg. Mai. Schickele, Die Beurteilung schwerer Blutungen unter der Geburt.

Munch, med. Wochenschr. 26. Mai. S. 1119.

126

Literatur - Verzeichnis.

Schönbek, A., Ein interessanter Fall von Eclampsia in graviditate und Missbildung der Frucht.

Centralbl. f. Gyn. 23. Mai. S. 707.

Slemons und Goldsborough, Die geburtshülfliche Bedeutung des Blut-drucks und sein

Verhältnis zur Arbeit des Herzens. Centralbl. f. Gyn. 23. Mai. S. 699.

Williamson, H., A case of intra-uterin $\theta$ death of the foetus occurring in six consecutive pregnancies: with observations upon the importance of the examination of the foetal tissues for spirochaeta pallida. Journ. of Obst. and Gyn. of the British Empire. Mai. S. 342.

Ziegenspeck, R., Zur Ätiologie der Retroflexio uteri puerperalis. Centralbl. f. Gyn. 6. Juni. S. 763.

Abortus. - Geburtshülfliche Operationen. - Operationen im

Wochenbett.

Golorní, C, Di un caso di mola carnosa-vescicolare-ematomatosa etc.

(Forts.) Lucina. 1. Mai. S. 70. Drake, F., Die Zangenentbindungen an der Göttinger Univ.-

Frauen-

klinik 1888-1907. Inaug.-Diss. Göttingen 1908. Ehrlich, H., Ein Beitrag zur Hebosteotomie.

Centralbl. f. Gyn. 16. Mai.

S. 651. Friedemann, E., Ein Fall von puerperaler Pyämie, geheilt durch Unter-

bindung der Beckenvenen. Centralbl. f. Gyn. 6. Juni. S. 761. Friedmann, M., Zur

Indikationsstellung für den künstlichen Abort wegen

psychischer Krankheit. Deutsche med. Wochenschr. 7.-14., 21. Mai.

S. 821 u. 873, 908. Fry, H., The management of labor in minor degrees of pelvic contraction, with special reference of the relative indications for abdominal cesarean section. A tabulated report of 18 cesarean

sections. Amer. Journ. of the med. Sciences. Mai. S. 704. Haim, E., Beitrag zur

Nierendekapsulation bei Eklampsie. Centralbl.

f. Gyn. 16. Mai. S. 666. Jereslaw, B., Indikationen zur künstlichen Unterbrechung der Schwanger- 
schaft. Inaug.-Diss. Freiburg. Mai. Kedarnath Das, Three cases of caesarean hysterectomy for stenosis of the vagina. Journ. of Obst. and Gyn. of the brit. Empire. Mai.

S. 351. Kownatzki, Geburtsstörung durch vaginale Fixation. Klassischer Kaiserschnitt. Munch, med. Wochenschr. 19. Mai. S. 1078. Kreisch, E., Sectio caesarea vaginalis bei Eklampsie. Munch, med.

Wochenschr. 2. Juni. S. 1183. Kroemer, P., Die Erfahrungen der Universitäts-Fraußnklinik an der $\mathrm{Kgl}$.

Charité über die Pubotomie. Berl. klin. Wochenschr. 1. Juni.

S. 1044. Lunckenbein, Der vaginale Kaiserschnitt in der Praxis. Munch, med.

Wochenschr. 9. Juni. S. 1229. Marx, H., Der kriminelle Abort. Berliner klin. Wochenschr. 18. Mai.

S. 965. Mehliss, M., Über die Berechtigung der prophylaktischen Wendung bei engem Beckon. Inaug.-Diss. Göttingen. 1908,

Literatur-Verzeichnis.

127

Nacke, Dammschutz. Centralbl. f. Gyn. 13. Juni. S. 795.

Peham, H., Zur Indikationsstellung bei der Pubotomie. Centralbl. f.

Gyn. 6. Juni. S. 768. Polano, 0., Kaiserschnitt und Lumbalanästhesie. Munch, med. Wochen-

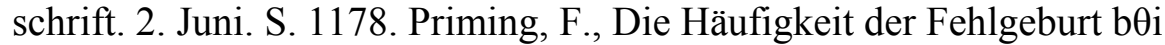

Zwillingsschwangerschaft.

Deutsche med. Wochenschr. 4. Juni. S. 1017. Spaeth, F., Ein Fall von cervikalem Kaiserschnitt. Centralbl. f. Gyn.

16. Mai. S. 654. Zangemeister, W., Eine selten $\beta$ Komplikation des Forceps bei Vorderhauptslage. Centralbl. f. Gyn. 16. Mai. S. 664. Ziegenspeck, R., Zum hohen Querstand der Gesichtslage und ihrer Behandlung mittels hoher Zange. Centralbl. für Gyn. 13. Juni.

S. 791.

Fötus. - Neugeborenes. Grüneberg, L., Zur Behandlung der hämorrhagischen Diathese beim N"eugeborenen. Munch, med. Wochenschr. 19. Mai. S. 1079. Heil, K., Lymphangioma cysticum thoracis als Geburtshindernis. Gyn.

Rundsch. H. 9. S. 330. Johnson, L., The necessity for the artificial feeding of infants. Amer. Journ. of Obst. and Dis. of Worn, and Childr. Mai. S. 671. Legge, S. C, Calcium chloride in melaena neonatorum. Brit. med.

Journ. 16. Mai. S. 1173. Nauwerck u. Flinzer, Paratyphus und Melaena des Neugeborenen. Munch, med. Wochenschr. 9. Juni. S. 1217. Northcote, A. B., Case of congenital abnormality of hands and feet.

Brit. med. Journ. 9. Mai. S. 1107.

Ektopische Schwangerschaft.

Horneman, Sv., Ein Fall von Graviditas extra-uterina mit lebender, ausgetragener Frucht.

Centralbl. f. Gyn. 16. Mai. S. 668.

Searle, E. A., A case of ectopic gestation with unusually early symptoms. Brit. med. Journ. 16.

Mai. S. 1162.

II. Gynäkologie.

Allgemeines.

Barret, W., The crime of gynecology. Amer. Journ. of Obst. and Dis. 
of Worn, and Childr. Mai. S. 617. delle Chiaje, S., Über ein Cytotoxin, welches die Fettentartung des

Eierstocks hervorruft. Centralbl. f. Gyn. 23. Mai. S. 705. Fromme u. Heynemann, Über die Hämolyse der Streptokokken. Berliner

klin. Wochenschr. 11. Mai. S. 919. Koblanck, Über nasale Reflexe. Deutsche med. Wochenschr. 11. Juni.

S. 1046. Kuttner, A., Die nasale Dysmenorrhoe. Deutsche med. Wochenschr.

11. Juni. S. 1050. Liepmann, W., Bakteriologie und Prognose. Berliner klin. Wochenschr.

1. Juni. S. 1046.

128

Literatur -Verzeichnis.

Mutter, E., Beitrag zur Frage der natürlichen Nutzstoffe in der Frauen-milch. Berliner klin.

Wochenschr. 1. Juni. S. 1058.

Osten, A., Untersucliungen über die Gerinnung des Blutes während der Menstruation. Inaug.-

Diss. Göttingen 1908.

Rosenkranz, E., Die Fulgurationsbehandlung der Krebse nach Keating-Hart. Berliner klin.

Wochenschr. 18. Mai. S. 957.

Savidge, E. G., The cancer problem. Med. Record. 2. Mai. S. 717.

Schückíng, Die rektale Instillation mit Natriumsaccharat-Kochsalzlösungen. Centralbl. f. Gyn. 16. Mai. S. 670.

Zacharias, P., Zwei Fälle von Tetanus nach gynäkologischen Operationen. Erwiderung. Munch, med. Wochenschr. 2. Juni. S. 1185.

Scheide.

Frankenstein, K.t Über vollkommenen Verschluss der Vagina. Deutsche

med. Wochenschr. 28. Mai. S. 969. Hinselmann, N., Beitrag zur Kenntnis der bösartigen

pigmentierten

Geschwülste der Vulva. Zeitschr. f. Geb. u. Gyn. Bd. 62. H. 1.

S. 34. Rath, W., Die Lappendammplastik und ihru Erfolge nach komplettem

Dammriss. Inaug.-Diss. Göttingen 1908.

Uterus und Operationen an demselben.

Ahreiner, G., Über die Blutungen bei der sogenannten chronischen Metritis.

Arch. f. Gyn. Bd. 88. H. 2. S. 372. Barbour, A. H. F., Das untere Uterinsegment. Zeitschr. f. Geb. und

Gyn. 62. Bd. H. 1. S. 91. Freeland Barbour: On the frozen sections by Bumm and Blumreich and by Zangemeister -- is there a lower uterina segment? II. Teil.

Journ. of Obst. and Gyn. of the brit. Emp. Mai. S. 315. Bland-Sutton, J., On one hundred consecutive hysterectomies for fibroids

attended with recovery: and observations on injuries of the uterus.

Journ. of Obst. and Gyn. of the brit. Emp. Mai. S. 328. Bossí, L. M., Der schneckenfòrmige Uterus. Zentralbl. f. Gyn. 16. Mai.

S. 662. Brodhead, G. L., Rupture of the uterus through the cesarean cicatrix.

Amer. Journ. of Obst. and dis. of worn, and child. Mai. S. 650. Kubo, T., Menorrhagia and metrorrhagia (uterine hemorrhage). Amer.

Journ of Obst. and dis. of worn, and childr. Mai. S. 675. Lockyer, C, Uterus unicornis, with haematometra and haematosalpinx. 
Journ. of Obst. and Gyn. of the brit. Emp. Mai. S. 359. Philipps, M. H., Uterus bicornis unicollis, Atresia of external os, absence

of vagina, retained menses, hysterectomy. Journ. of Obst. and Gyn.

of the brit. Emp. Mai. S. 355. Uhl, G., Stumpfkarzinome nach supravaginaler Amputation des

myoma-

tösen Uterus. Inaug.-Diss. Strassburg. April. Weishaupt, E., Zur Lehre von der Endometritis und der Bedeutung

der Plasmazellen bei pathologischen Gewebsreaktionen (Entzündung).

Zeitschr. f. Geb. und Gyn. 62. Bd. H. 1. S. 52.

Literatur -Verzeiehnis.

129

Tuben. - Ovarien. - Lígamente.

Bircher, E., Kombination von Ovarialkarzinom $\theta n$ mit anderen Ge-schwülsten. Arch. f. Gyn. Bd.

85. H. 2. S. 435.

Iwase, Y., Über primäre Chorioepitheliome des Ovariums. Arch. f. Gyn. Bd. 85. H. 2. S. 414.

Krusen, W., Report of 2 cases: A) Myxosarcoma of the round ligament. B) Fibroma of the

anterior abdominal wall. Amer. Journ. of Obst. and dis. of worn, and child. Mai. S. 666.

Kworostansky, P., Endotheliom des Ovariums und der Tube. Arch. f. Gyn. Bd. 85. H. 2. S. 355.

Lajos, Góth, Bedrohliche innere Blutung aus einem Perithelioma ovarii. Laparotomie. Heilung.

Zeitschr. f. Geb. und Gyn. 62. Bd. 1. H. S. 26.

Peterson, R., Preservation of the ovaries entire or in part in supra-vaginal or panhysterectomy.

Amer. Journ. of Obst. and dis. of worn, and childr. Mai. S. 633.

Ries, E, Vater-Pacinische Körperchen in der Tube. Zeitschr. f. Geb. und Gyn. 62. L·u. H. 1. S.

100.

Sitzenfrey, A., Beiderseitig’v Hydrosalpinx mit voUständig durchgängigem Tubenlumen,

offenem abdominalen und uterinen Tubenostium bei Pseudomyxoma peritonei. Gyn. Rundschau. H. 9. S. 321.

Wack, 0., Ein primäres Sarkom des Ligament, latum uteri. Inaug.-Diss. Freiburg. Mai.

Bauchhöhle. - Bauchschnitt. - Scheidenbauchschnitt.

Jerusalem, M., Einiges über Nachbehandlung Laparotomierter. Munch.

med. Wochenschr. 26. Mai. S 1130. Rosenthal, B., Zur Diagnose und Operation der

intraabdominellen Lymph-

angiome. Zentralbl, f. Gyn. 30. Mai. S. 731. Wilcox, S. F., Inunction of jodoform in tuberculous peritonitis. Med.

Record. 2. Mai. S. 735.

Narkose. - Anti- und Asepsis.

Mayer, K., Skopolamin-Morphium bei Geburten. Centralbl. f. Gynäkol.

23. Mai. S. 689. Sïeber, H., Beitrag zur Skopolamin-Morphiumnarkose in der Gynäkologie.

Centralbl. f. Gyn. 13. Juni. S. 785.

Harnorgane. - Fisteln.

Arndt, C, Okklusivpessar als Fremdkörper in der Blase. Centralbl. f.

Gyn. 23. Mai. S. 716. Gurrie, O. J., Five cases of vesico-vaginal fistula. Brit. med. Journ.

9. Mai. S. 1108. Teller, R., Über Incontinentia urina $\theta$ bei Spaltbildung der weiblichen

Urethra (sog. weibliche Hypospadie) und ihre operative Behandlung.

Zeitschr. f. Geb. u. Gyn. 62. Bd. H. 1. S. 1. v. Valenta, A., Kolpokleisis wegen handtellergrossen Defektes der Blase -- 
post partum -, nachfolgende Ovariotomie. Heilung. Gyn. Rundsch.

H. 9. S. 329. Monatsschrift fur Geburtshülfe u. Gynäkologie. Bd. XXVIII. Heft 1. 9

130

Literatur-Verzeichnis.

Mamma.

Oroll, Ph., Über diffuse Hypertrophie der weiblichen Brustdrüse. Inaug.-

Diss. München. Mai. Kayser, F, Achselhöhlenbrüste bei Wöchnerinnen. Arch. f. Gyn. Bd. 85.

H. 2. S. 459.

Darm. - Hernien.

Becker, P., Myome des Mastdarms. Arch. f. Gyn. Bd. 85. H. 2. S. 427. Port, K., Die Grasersche

Operation grosser Nabel- und Bauchbrüche. Munch, med. Wochenschr. 9. Juni. S. 1231.

Technisches und Akiurgie.

Benckiser und Krumm, Zur Karzinombehandlung nach Dr. d $\theta$ Keating-Hart. Deutsche med.

Wochenschr. 1-1 Mai. S. 885.

Hoffmann, J, Ein neues Instrument zur Behandlung der SchlӨimhaut der Cervix uteri. Centralbl. f. Gyn. 30. Mai. S. 744.

Rudolph, J, Die Federcurette zur Vermeidung der Perforatio uteri. Centralbl. f. Gyn. 16. Mai. S. 672.

Stähler, F., Vßreinigung von Instrumentenkocher und Instrumententisch. Centralbl. f. Gyn. 23.

Mai. S. 716.

Verantwortlicher Redakteur: A. Martin in Berlin. 